\title{
Dissociative electron attachment and electron energy-loss spectra of phenyl azide
}

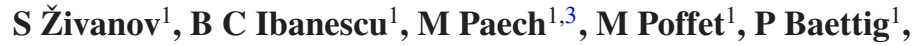 \\ A-C Sergenton ${ }^{1}$, S Grimme $^{2}$ and M Allan ${ }^{1}$ \\ ${ }^{1}$ Department of Chemistry, University of Fribourg, chemin du Musée 9, 1700 Fribourg, \\ Switzerland \\ ${ }^{2}$ Organisch-Chemisches Institut der Universität Münster, Corrensstrasse 40, 48149 Münster, \\ Germany
}

\begin{abstract}
Electron-induced chemistry-dissociative electron attachment (DEA)—was studied for phenyl azide. The major fragment corresponded to the loss of $\mathrm{N}_{2}$ and formation of the phenylnitrene anion. This process has an onset already at zero kinetic energy of the incident electron and is interpreted as proceeding via the $\mathrm{A}^{\prime \prime} \pi^{*}$ electronic ground state of the phenyl azide anion. Other fragments, $\mathrm{N}_{3}^{-}$and $\mathrm{CN}^{-}$, were observed at higher energies and interpreted as proceeding via low-lying shape resonances or higher lying core-excited resonances. The interpretation of the dissociative attachment spectra was supported by an investigation of the excited electronic states of neutral phenyl azide by electron energy-loss spectroscopy and DFT/MRCI calculations, and a study of shape and core-excited resonances of the phenyl azide anion by means of electron transmission spectroscopy and of cross sections for vibrational and electronic excitation by electron impact. Interesting parallels and differences are found by comparing DEA of phenyl and benzyl azides with the corresponding chloro compounds.
\end{abstract}

\section{Introduction}

Interesting applications in electron-beam lithography [1] renewed the interest in electroninduced chemical processes. At subionization energies these processes can proceed either via electronic excitation of the target or, particularly at very low, subexcitation, energies, by way of dissociative electron attachment (DEA) [2]. The latter is particularly important because in a real situation of electron-beam processing or radiation-induced damage a high energy

3 Present address: Leibniz Universität Hannover, Institut für Theoretische Physik, 30167 Hannover, Germany. 


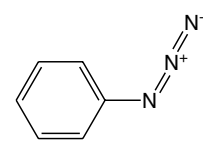

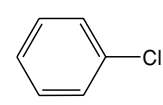

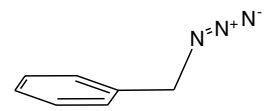

3

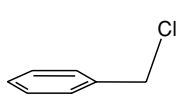

4

Figure 1. Structures of phenyl azide (1), chlorobenzene (2), benzyl azide (3) and benzyl chloride (4). The molecules $\mathbf{1}$ and $\mathbf{2}$ are planar, the $\mathrm{C}-\mathrm{N}$ and $\mathrm{C}-\mathrm{Cl}$ bonds in $\mathbf{3}$ and $\mathbf{4}$ point out of the plane of the phenyl ring.

primary electron (or $\gamma$ photon) impinging on a material or on biological tissue leads to a 'shower' of secondary electrons with low, subexcitation energies. For understanding the real situation it is also important to know where the entirely slowed-down electrons will be finally trapped, and whether they will cause a chemical change while being trapped. Dissociative electron attachment processes generally have an energetic threshold, given by the difference of the bond dissociation energy and the electron affinity of the fragment. The threshold is well above zero electron energy for most molecules, and very slow electrons then cannot induce chemical change. Exceptions are primarily halogenated compounds, where the large electron affinity of the halogen, or of a halogen containing fragment, leads to DEA at zero electron energy [3]. This property makes halogenated compounds useful as electron scavengers, for example in matrix isolation studies [4]. In this work [5] we investigate phenyl azide $\mathbf{1}$ (figure 1), an alternative to halogen compounds in the sense that DEA at very low energy is driven by the high stability of the neutral fragment. The results are in an interesting contrast to those obtained for the related benzyl azide 3 by Illenberger et al [6] and the halogen compounds chlorobenzene 2 and benzyl chloride 4 .

\section{Methods}

\subsection{Experiment}

The dissociative electron attachment spectrometer used to measure the yield of mass-selected stable anions as a function of electron energy was described previously [7-9]. It employs a magnetically collimated trochoidal electron monochromator to prepare a beam of quasimonoenergetic electrons, which is directed into a target chamber filled with a quasi-static sample gas. Fragment anions are extracted at $90^{\circ}$ by a three-cylinder lens and directed into a quadrupole mass spectrometer. The spectra were calibrated on the onset of the $\mathrm{O}^{-} / \mathrm{CO}_{2}$ signal. The electron current was several nanoamperes and the resolution was about $70 \mathrm{meV}$. Electron energy-loss spectra were recorded with a trochoidal electron spectrometer [10, 11]. The photoelectron spectrum was recorded with a modified Perkin Elmer PS18 HeI photoelectron spectrometer.

\subsection{Computational details}

For the computation of singlet and triplet state energies and wavefunctions of phenyl azide, we used the combined density functional theory/multi-reference configuration interaction (DFT/MRCI) method [12]. This approach was shown to yield excellent electronic spectra of organic molecules at reasonable computational expense [13-16]. As AO basis we employ the aug-cc-VTZ $(\mathrm{C}, \mathrm{N})$ and cc-pVTZ $(\mathrm{H})$ sets of Dunning $[17,18]$ but with discarded f-function on carbon and nitrogen. The B3LYP/TZVP [19-21] optimized ground state geometry 


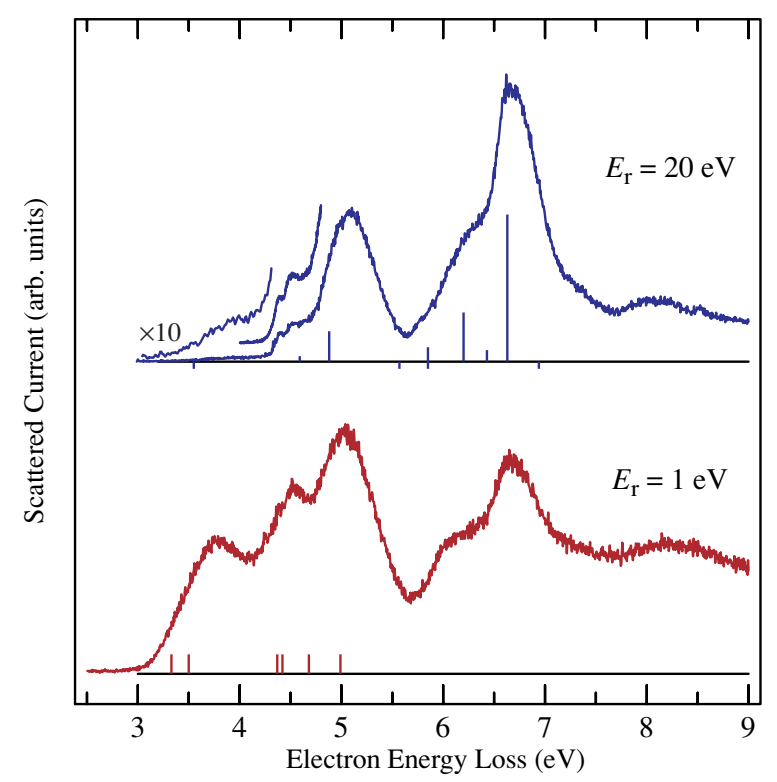

Figure 2. Electron energy-loss spectra of phenyl azide. Top: conditions emphasizing singletsinglet transitions, bottom: conditions emphasizing singlet-triplet transitions. Bars below the top spectrum indicate calculated transition energies and dipole intensities (transitions with an oscillator strength of less than $f=0.02$ are indicated by a tick below the baseline for clarity). Bars below the bottom spectrum indicate calculated singlet-triplet transition energies.

$\left(\mathrm{C}_{\mathrm{s}}\right.$ symmetry) is used and thus all calculated excitation energies correspond to vertical transitions. The configuration state functions (CSFs) in the MRCI expansion are built up from Kohn-Sham (KS) orbitals, optimized for the dominant closed shell determinant of the electronic ground state employing the BH-LYP $[22,23]$ functional. All 44 valence electrons were correlated in the MRCI runs and the eigenvalues and eigenvectors of 16 singlet and 8 triplet states were determined. The initial set of reference CSFs was generated automatically in a complete active space type procedure (including all single and double excitations from the four highest occupied molecular orbitals in the KS determinant to the six lowest virtual orbitals) and was then iteratively improved. The MRCI expansion was kept moderate by extensive configuration selection. The variational space was spanned by 50-80000 energy-selected single and double excitations (about 170000 singlet and 150000 triplet CSFs in each of the irreducible representations) generated from about 200 (singlet) and 140 (triplet) reference configurations.

\section{Results and discussion}

\subsection{Electronic structure of neutral phenyl azide}

Figure 2 shows electron energy-loss spectra recorded with the trochoidal electron spectrometer once at the low electron energy of $1 \mathrm{eV}$-yielding a spectrum emphasizing singlet-triplet transitions - and once at the higher electron energy of $20 \mathrm{eV}$-emphasizing dipole-allowed transitions. The lowest excited state with a very low oscillator strength $(f=0.0007)$ has been predicted to be a $\mathrm{a}^{\prime \prime 1}\left(n, \pi^{*}\right)$ state at $3.55 \mathrm{eV}$ by the DFT/MRCI calculation. The 


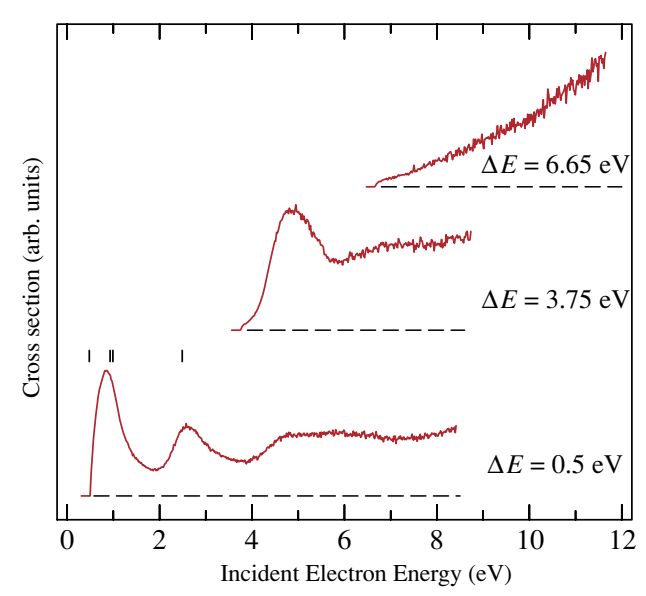

Figure 3. Excitation functions of phenyl azide. Bottom: pure vibrational excitation, centre: excitation of a triplet state, top: excitation of a dipole-allowed singlet-singlet transition. Vertical bars indicate the attachment energies estimated from the virtual orbital energies using the empirical scaling relation of Chen and Gallup [27].

upper spectrum shows a weak shoulder with an onset at about this energy which is likely to correspond to this state, although a contribution from a triplet state cannot be excluded. A more intense structured shoulder appears in the upper spectrum at $4.5 \mathrm{eV}$, in good agreement with the calculation which predicts a weak $\mathrm{a}^{\prime}$ band $(f=0.039)$ at $4.59 \mathrm{eV}$. A more intense band appears at $5.07 \mathrm{eV}$, in satisfactory agreement with the calculation which predicts a fairly strong a' transition at $4.88 \mathrm{eV}$. A shoulder at about $6.2 \mathrm{eV}$ in the measured spectrum agrees well with the three calculated states at 5.85, 6.20 and $6.43 \mathrm{eV}$ (all $\mathrm{a}^{\prime}$ ). The most intense transition $\left(\mathrm{a}^{\prime}\right)$ is calculated at $6.63 \mathrm{eV}$ with $f=1.02$, in a very good agreement with the highest peak in the spectrum, observed at $6.65 \mathrm{eV}$. The weak transition calculated at $5.57 \mathrm{eV}$ has a wavefunction spatially more extended than the electronic ground state, indicating a Rydberg character. Triplet states are observed at 3.78, 4.52 and $5.03 \mathrm{eV}$ and the calculation is consistent with the observed spectrum.

\subsection{Electronic structure of phenyl azide anion}

The shape resonances, short-lived states of the anion where the incident electron is temporarily captured in an empty orbital of the target, can be determined by means of the electron transmission spectroscopy [24] or as an enhancement of the cross section for vibrational excitation of the target $[11,25]$. The vibrational excitation cross section is shown in figure 3 and exhibits two bands, at 0.85 and $2.6 \mathrm{eV}$. The same two bands appear also in the transmission spectrum, which we recorded, but do not show here. Four resonances are expected in phenyl azide at low energies. They can be represented as combinations of the two degenerate $\pi^{*}$ resonances of the benzene ring (at $1.4 \mathrm{eV}$ in benzene [24]) and two $\pi^{*}$ resonances of the azide group. A useful way of estimating the energies of shape resonances in large molecules where scattering calculations are impractical is the use of the scaled Koopmans theorem [27]. This method, applied to HF 6-31G* virtual orbitals (at geometry calculated with DFT B3LYP), yields the values $0.48,0.93,0.99$ and $2.49 \mathrm{eV}$, indicated by vertical bars in figure 3 . The attachment energies estimated in this way rationalize very well the observation when it is taken into account that the threshold for vibrational excitation with $\Delta E=0.5 \mathrm{eV}$ is at 
$0.5 \mathrm{eV}$, and the peak at the predicted energy of $0.48 \mathrm{eV}$ cannot appear directly. The very steep onset of the vibrational cross section at the threshold is a strong indication of a resonance at or slightly below $0.5 \mathrm{eV}$. The $\mathrm{A}^{\prime \prime}$ resonances predicted at 0.48 and $2.49 \mathrm{eV}$ are due to temporary occupation of the in-phase and out-of-phase combinations of the $\pi^{*}$ MOs of the phenyl ring and the $\mathrm{N}_{3}$ group, respectively. The $\mathrm{A}^{\prime \prime}$ resonance predicted at $0.99 \mathrm{eV}$ is due to temporary occupation of the second $\pi^{*} \mathrm{MO}$ of the phenyl ring, which has a node at the position where the $\mathrm{N}_{3}$ group is attached, and is therefore at nearly the same energy as in benzene. The $\mathrm{A}^{\prime}$ resonance predicted at $0.93 \mathrm{eV}$ is due to temporary occupation of the $\pi^{*} \mathrm{MO}$ of the $\mathrm{N}_{3}$ group which lies in the plane of the molecule and does not conjugate with the phenyl $\pi^{*}$ MOs (that is, from the point of view of the entire molecule it is a $\sigma^{*} \mathrm{MO}$ ). Note that the estimated lowest attachment energy of $0.48 \mathrm{eV}$ refers to a vertical transition and the anion in its relaxed geometry is nearly certainly bound. The step at $4.7 \mathrm{eV}$ in the lowest curve of figure 3 is more difficult to associate with a specific shape resonance, but could be a higher lying $\pi^{*}$ resonance located on the benzene ring, found at $4.8 \mathrm{eV}$ in benzene [24].

Peaks in the cross sections for the excitation of electronic states are generally indications for (valence) core-excited resonances. The peak at $4.87 \mathrm{eV}$ in the cross section for the excitation of the lowest triplet excited state is thus an indication of a core-excited resonance. The cross section in the topmost trace in figure 3 is a nearly linear function of energy and characteristic for a dipole allowed transition, in agreement with the prediction of the DFT/MRCI calculation discussed above.

Feshbach resonances with a hole in a valence orbital and two electrons in a diffuse Rydberg-like orbital have been shown to be often responsible for dissociative electron attachment both in small [37] and in large molecules [28]. These resonances affect the elastic and the vibrational excitation cross sections only weakly and can be identified in these channels only when they have very narrow vibrational features, which is not the case for phenyl azide. Their energies can be estimated from the ionization energies, determined by photoelectron spectroscopy-because the electrons in the diffuse orbitals penetrate nominally only weakly into the cationic core, their binding energy is only relatively weakly dependent on the nature of this core, it is around $4-4.5 \mathrm{eV}$ in larger polyatomic molecules. This method is particularly useful in predicting the energies (and Franck-Condon band profiles) of Feshbach resonances in larger molecules where the photoelectron bands and consequently also the Feshbach resonances have relatively broad Franck-Condon profiles without resolved vibrational structure [28]. The photoelectron spectrum has been reported by Bastide and Maier [29]. The lowest vertical ionization energy is $8.72 \mathrm{eV}$. It corresponds to ionization from the $\pi^{*}$ HOMO orbital which extends over both the phenyl ring and the azide group and permits a prediction of a Feshbach resonance around 4.2-4.7 eV.

Finally, core-excited resonances with valence-excited parent states have often been identified to be responsible for DEA, particularly in molecules with $\pi$ and $\pi^{*}$ orbitals and thus low-lying valence-excited states. These resonances generally energetically coincide with low-lying singlet valence-excited states $[30,31]$. They could be expected at the energies of the singlet excited states seen in the upper energy-loss spectrum in figure 2.

\subsection{Dissociative electron attachment}

The large DEA cross section for phenylnitrene anion production from $\mathbf{1}$ has already been used to prepare this anion in large quantities for the measurement of its photoelectron spectrum [32] and for a variable-wavelength photodetachment study [33]. The latter determined the EA of the triplet phenylnitrene as $1.43 \mathrm{eV}$, that of the singlet phenylnitrene as $2.22 \mathrm{eV}$. 


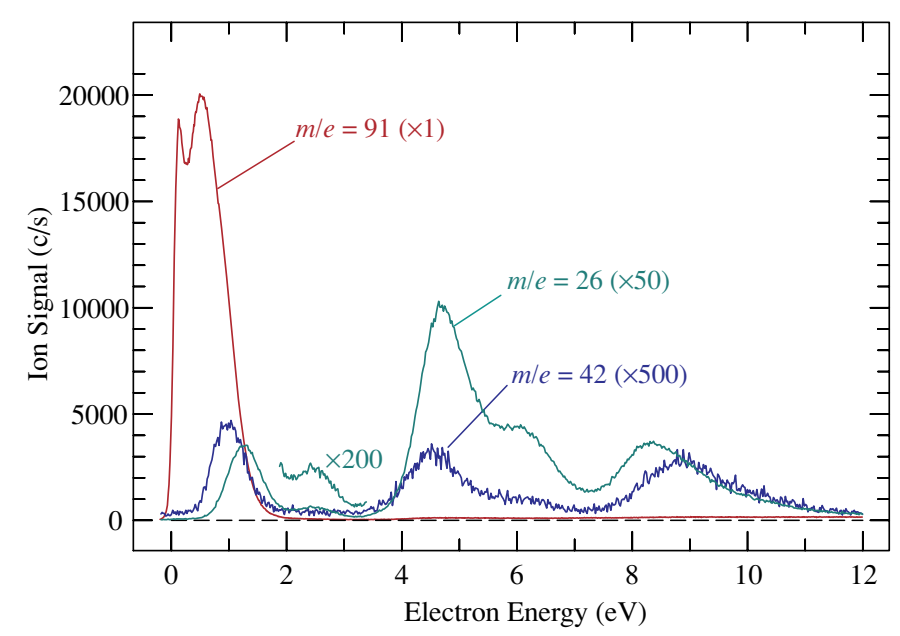

Figure 4. Dissociative electron attachment spectra of phenyl azide.

Our dissociative electron attachment spectra are shown in figure 4. The spectra are not in absolute units but relative intensities are indicated by the count rates. The most intense signal is mass 91, the phenylnitrene anion, resulting from a loss of $\mathrm{N}_{2}$. The gas-phase ion chemistry of the phenylnitrene anion has been investigated with the observation that the anion does behave like a nitrene radical anion, rather than some rearranged species [34] and we therefore assume that also in our case the mass 91 signal corresponds to phenyl nitrene and not to any rearranged ion. The phenylnitrene anion signal has a vertical onset at zero incident energy. It can be assigned to dissociation via the lowest resonance with a predicted vertical attachment energy of $0.48 \mathrm{eV}$, but whose Franck-Condon profile most likely extends down to zero incident energy. A rise of signal with electron energy decreasing towards zero $\mathrm{eV}$ is often observed in DEA. It is also observed in the present case and is presumably a consequence of the fact that autodetachment rate generally decreases with decreasing electron energy and the competing dissociation channel thus gains in importance. The subsequent peak of the cross section at about $0.5 \mathrm{eV}$ could be due to the peak of the Franck-Condon envelope (vertical attachment) of the lowest shape resonance.

Mass 26 could be either the cyanide anion $\mathrm{CN}^{-}$or the vinylidene anion $\mathrm{C}_{2} \mathrm{H}_{2}^{-}$. Measurement of the perdeuterated compound phenyl azide- $\mathrm{d}_{5}$ has also yielded mass 26 , proving that the fragment is $\mathrm{CN}^{-}$. It could be generated in a two-step mechanism where $\mathrm{N}_{2}$ is lost first, and the resulting hot phenylnitrene anion further decomposes into $\mathrm{CN}^{-}$and a cyclopentadienyl radical $c-\mathrm{C}_{5} \mathrm{H}_{5}$. The onset of the first peak in the mass 26 spectrum probably coincides with the energetic threshold for the process. The next band, at $2.48 \mathrm{eV}$, corresponds doubtlessly to the shape resonance observed at $2.60 \mathrm{eV}$ in the vibrational excitation spectrum in figure 3, and in the transmission spectrum. Three higher lying bands are observed at 4.68, 6.04 and $8.35 \mathrm{eV}$. Their assignment is not certain. The lowest band, at $4.68 \mathrm{eV}$, is energetically close to the expected position of the lowest Feshbach resonance, expected around this energy from the PE-spectrum. It is also quite close to the core-excited resonance seen at $4.87 \mathrm{eV}$ in the triplet excitation cross section in figure 3 , so that both assignments are possible. The assignment of the 6.04 and $8.35 \mathrm{eV}$ bands is puzzling - they do not have a counterpart in the photoelectron spectrum, excluding an assignment to Feshbach resonances associated with the higher ionization energies. An assignment to valence core-excited resonances of the type 
Table 1. Calculated threshold energies, obtained as a difference of total energies of the products and the target at $0 \mathrm{~K}$, corrected for the zero point vibrational energy. The DFT B3LYP/6$311+\mathrm{G}(2 \mathrm{df}, 2 \mathrm{p})$ model [35] was used.

\begin{tabular}{lc}
\hline Reaction & $E_{\mathrm{thr}}$ \\
\hline $\mathrm{e}^{-}+\mathrm{C}_{6} \mathrm{H}_{5} \mathrm{~N}_{3} \rightarrow \mathrm{C}_{6} \mathrm{H}_{5} \mathrm{~N}^{-}+\mathrm{N}_{2}$ & $-1.5 \mathrm{eV}$ \\
$\mathrm{e}^{-}+\mathrm{C}_{6} \mathrm{H}_{5} \mathrm{~N}_{3} \rightarrow \mathrm{C}_{6} \mathrm{H}_{5}+\mathrm{N}_{3}^{-}$ & $+0.9 \mathrm{eV}$ \\
$\mathrm{e}^{-}+\mathrm{C}_{6} \mathrm{H}_{5} \mathrm{CH}_{2} \mathrm{~N}_{3} \rightarrow \mathrm{C}_{6} \mathrm{H}_{5} \mathrm{CH}_{2}+\mathrm{N}_{3}^{-}$ & $-0.4 \mathrm{eV}$ \\
\hline
\end{tabular}

discussed by Khvostenko et al [31] also appears improbable in view of the singlet excitation energies shown in figure 2.

Mass 42 is the azide anion $\mathrm{N}_{3}^{-}$. The first band in the yield of this fragment is probably due to the $\mathrm{A}^{\prime}$ resonance predicted at $0.99 \mathrm{eV}$ (see section 3.2 and the discussion below). The energies of the higher lying bands are similar to those observed with mass 26 .

Not having found suitable experimental thermochemical data we retorted to calculations to determine threshold energies for three processes relevant to the present work and the results are listed in table 1. The large calculated exothermicity of the loss of $\mathrm{N}_{2}$ from the present compound is consistent with the observed signal rise at $0 \mathrm{eV}$. The calculated threshold energy for the formation of $\mathrm{N}_{3}^{-}$from phenyl azide is consistent with the signal onset in figure 4 $(0.65 \mathrm{eV}$ at $50 \%$ of peak intensity) within the reliability which may be expected from this type of calculation. The formation of $\mathrm{N}_{3}^{-}$from benzyl azide is calculated to be exothermic, in agreement with the steep signal onset near $0 \mathrm{eV}$ observed by Illenberger et al [6].

The azide anion $\mathrm{N}_{3}^{-}$is a pseudohalide [6], the $\mathrm{N}_{3}$ radical behaves chemically like a halogen atom. Interesting parallels and differences can consequently be recognized by comparing the DEA of phenyl and benzyl azide with the corresponding halides, for example the chlorobenzene and benzyl chloride shown in figure 1 . The following observations were made in the chloro compounds ([9, 26, 36, 38] and references therein).

- The loss of the halide anion is allowed even in the geometry of the ground state of the non-planar benzyl chloride. DEA consequently proceeds with a large cross section and with substantial release of kinetic energy [36, 37].

- In chlorobenzene dissociation is symmetry forbidden in the planar geometry of the neutral molecule and proceeds with a smaller cross section. This is because, although dissociation of the chlorobenzene anion is allowed through vibronic coupling and ensuing symmetry lowering, and proceeds without any activation barrier [38], the anion has to distort from the planar geometry in the initial stages of the dissociation and the slope of the potential hypersurface along the reaction coordinate is consequently initially smaller in the phenyl compound than in the benzyl compound.

These effects are accentuated by the fact that the energetic threshold for DEA is lower in the benzyl compounds than in the phenyl compounds because the more extended $\pi$-electron system makes the benzyl radical more stable than the phenyl radical.

Similarly, there is a dramatic difference between the DEA behaviour of phenyl and benzyl azides. Benzyl azide is non-planar [39] and the loss of the pseudohalide $\mathrm{N}_{3}^{-}$is allowed in the geometry of the neutral target. In view of this and the low-lying energetic threshold (table 1) it is not surprising that it is the major channel [6], in an analogy to the efficient $\mathrm{Cl}^{-}$loss from benzyl chloride.

Vertical attachment of a slow electron to phenyl azide leads to a $\mathrm{A}^{\prime \prime}$ planar ground state of the anion whose electronic wavefunction is antisymmetric with respect to the molecular plane 
(section 3.2). Loss of $\mathrm{N}_{3}^{-}$is consequently symmetry forbidden in the planar geometry because both the phenyl radical $\left({ }^{2} \mathrm{~A}_{1}\right)$ and $\mathrm{N}_{3}^{-}\left({ }^{1} \Sigma_{\mathrm{g}}\right)$ are symmetric with respect to the molecular plane. This dissociation is allowed from the $\mathrm{A}^{\prime}$ resonance predicted at $0.99 \mathrm{eV}$ (section 3.2), however.

The qualitative interpretation of the $\mathrm{C}_{6} \mathrm{H}_{5} \mathrm{~N}^{-}+\mathrm{N}_{2}$ dissociation channel at low energy is less straightforward. The phenylnitrene anion is assumed to have a ${ }^{2} \mathrm{~B}_{2}$ ground state, symmetric with respect to the molecular plane, with the singly occupied MO being a p-type nonbonding orbital in the molecular plane [32]. There is a low-lying ${ }^{2} \mathrm{~B}_{1}$ excited state, antisymmetric with respect to the molecular plane, with a singly occupied $\pi$ orbital, estimated to lie about $10 \mathrm{kcal} \mathrm{mol}^{-1}(0.43 \mathrm{eV})$ above the ground state [32]. The dissociation into the excited state of phenylnitrene anion is thus symmetry allowed in the planar geometry, whereas the dissociation into the ground state is not. The close proximity of the excited state will enhance vibronic coupling, however, and the dissociation can be expected to proceed quickly through symmetry lowering, in agreement with observations.

\section{Conclusions}

Dissociative electron attachment in phenyl azide is driven both by the high stability of the $\mathrm{N}_{2}$ fragment and by the sizeable electron affinity of the nitrene fragment. As a consequence, a very strong signal for the nitrene anion (loss of $\mathrm{N}_{2}$ ) is obtained by incident electrons already in the very low energy range $0-1 \mathrm{eV}$, where DEA proceeds via the lowest shape resonance. Weaker $\mathrm{CN}^{-}$and $\mathrm{N}_{3}^{-}$signals are observed peaking at $0.8 \mathrm{eV}$ and $1.2 \mathrm{eV}$ where the DEA proceeds via higher lying shape resonances. This is in an interesting contrast to the behaviour of benzyl azide, where the $\mathrm{N}_{3}^{-}$channel is by far dominant [6]. The differences can be understood in terms of the threshold energies and symmetry considerations. More DEA bands are observed at higher energies, 4-10 eV, the energy range of core-excited resonances.

\section{Acknowledgment}

This research is part of project No. 200020-105226/1 of the Swiss National Science Foundation.

\section{References}

[1] Schmelmer U, Jordan R, Geyer W, Eck W, Gölzhäuser A, Grunze M and Ulman A 2003 Angew. Chem. Int. Ed. Engl. 42559

[2] Illenbergger E and Momigny J 1992 Gaseous Molecular Ions (New York: Springer)

[3] Christophorou L G and Olthoff J K 2003 Fundamental Electron Interactions with Plasma Processing Gases (Dordrecht: Kluwer Academic)

[4] Bally T 2004 Reactive Intermediate Chemistry ed R A Moss, M S Platz and M Jones Jr (New York: Wiley)

[5] Paech M 2000 Diploma Thesis, Fribourg (unpublished)

[6] Illenberger E, Comita P B, Brauman J I, Fenzlaff H P, Heni M, Heinrich N, Koch W and Frenking G 1985 Ber. Bundessges. Phys. Chem. 891026

[7] Stepanović M, Pariat Y and Allan M 1999 J. Chem. Phys. 11011376

[8] Dressler R and Allan M 1985 Chem. Phys. 92449

[9] Bulliard C, Allan M and Haselbach E 1994 J. Phys. Chem. 9811040

[10] Allan M 1982 Helv. Chim. Acta 652008

[11] Allan M 1989 J. Electron Spectrosc. Relat. Phenom. 48219

[12] Grimme S and Waletzke M 1999 J. Chem. Phys. 1115645

[13] Tomic K, Tatchen J and Marian C M 2005 J. Phys. Chem. A 1098410

[14] Diedrich C and Grimme S 2003 J. Phys. Chem. A 1072524

[15] Tatchen J, Waletzke M, Marian C M and Grimme S 2001 Chem. Phys. 264245 
[16] Parusel A B J and Grimme S 2001 J. Porphyrins Phthalocyanines 5225

[17] Kendall R A, Dunning T H and Harrison R J 1992 J. Chem. Phys. 966796

[18] Dunning T H 1989 J. Chem. Phys. 901007

[19] Becke A D 1993 J. Chem. Phys. 985648

[20] Stephens P J, Devlin F J, Chablowski C F and Frisch M J 1994 J. Phys. Chem. 9811623

[21] Schäfer A, Huber C and Ahlrichs R 1994 J. Chem. Phys. 1005829

[22] Becke A D 1993 J. Chem. Phys. 981372

[23] Lee C, Yang W and Parr R G 1988 Phys. Rev. B 37785

[24] Jordan K D and Burrow P D 1987 Chem. Rev. 87557

[25] Schulz G J 1973 Rev. Mod. Phys. 45423

[26] Stricklett K L and Burrow P D 1986 J. Phys. B: At. Mol. Phys. 194241

[27] Chen D and Gallup G A J 1990 J. Chem. Phys. 938893

[28] Skalický T and Allan M 2004 J. Phys. B: At. Mol. Opt. Phys. 374849

[29] Bastide J and Maier J P 1976 J. Electron Spectrosc. Relat. Phenom. 9307

[30] Dressler R A, Allan M and Tronc M 1987 J. Phys. B: At. Mol. Phys. 20393

[31] Khvostenko V I, Vorob'yov A S and Khvostenko O G 1990 J. Phys. B: At. Mol. Opt. Phys. 231975

[32] Travers M J, Cowles D C, Clifford E P and Ellison G B 1992 J. Am. Chem. Soc. 1148699

[33] Richard N, McDonald R N, Stephen J and Davidson S J 1993 J. Am. Chem. Soc. 11510857

[34] Drzaic P S and Brauman J I 1984 J. Am. Chem. Soc. 1063443

[35] Frisch M J et al 2004 Gaussian 03 (Wallingford CT: Gaussian) (rev. C.01)

[36] Dressler R, Allan M and Haselbach E 1985 Chimia 39385

[37] Stricklett K L, Chu S C and Burrow P D 1986 Chem. Phys. Lett. 131279

[38] Skalický T, Chollet C, Pasquier N and Allan M 2004 Phys. Chem. Chem. Phys. 43583

[39] Santos J P, Costa M L, Olariu R I and Parente F 2006 Eur. Phys. J. D 39379 